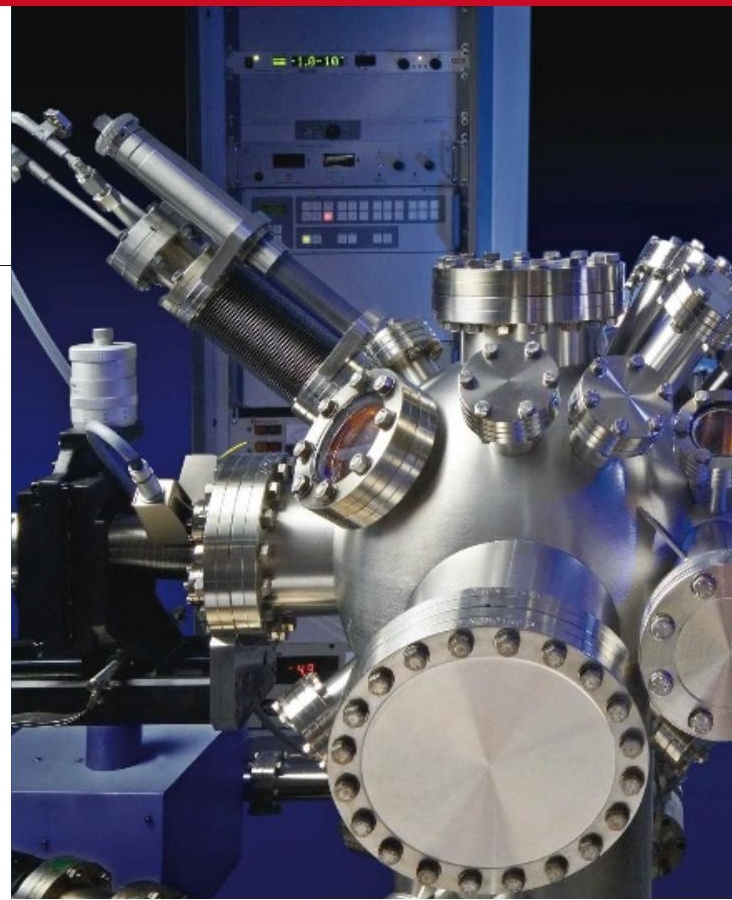

Peter Main, director of science and education at the UK Institute of Physics, says there is real concern about a shift from curiosity-driven research to applied programmes. "The real danger is that if you're not careful you end up funding lower-quality research simply because it is related to a particular topic," he says.

The EPSRC was given a budget increase of $18.6 \%$ by the government last year, but the extra money has been eaten up by inflation and its commitment to paying the full costs of research, which include maintaining equipment and building infrastructure. Rather than

\title{
String theorists hope to classify the cosmos
}

Physicists' search for a theory of everything is entering territory more familiar to biologists: taxonomy. A small team of theorists is meeting in Tucson, Arizona, in April to discuss how to classify the billions upon billions of different possible universes created by string theory, which describes fundamental particles and forces as vibrating strings.

"String theory is notorious for having a lot of solutions," says Keith Dienes, a physicist at the University of Arizona in Tucson. "We are having this big kick-off meeting to try and organize ourselves," he says. The String Vacuum Project aims to begin placing the various solutions into broad categories. If it works, then theorists may finally begin honing down the version of the theory that best fits with the cosmos.

But that's a pretty big if. There's no guarantee that the different possibilities will be easy to classify, or that the solution matching our Universe will be easy to find, according to Nathan Seiberg, a string theorist at the Institute for Advanced Study in Princeton, New Jersey. "I wouldn't hold my breath," he says, "but it is possible."

String theory is beloved by theorists because it is one of the few paradigms that promises to merge quantum mechanics, which explains the behaviour of particles on a subatomic level, with general relativity, which describes how gravity shapes large entities such as galaxies. To do so, the theory uses 10 or 11 dimensions of space-time. To make the theory resemble the physical world, theorists get rid of the extra dimensions by folding them up.

Like a sheet of paper, there are a nearly infinite number of ways to fold the extra dimensions, and each leads to a different 'vacuum' or

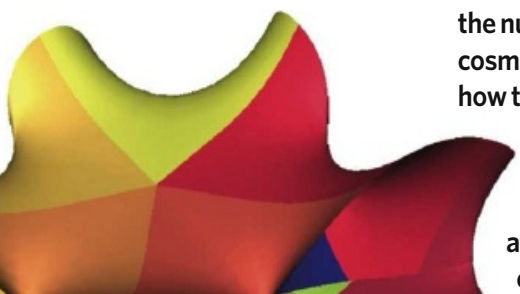
os, can all vary according to how the extra dimensions are folded. The number of possibilities has been steadily rising in recent years and current sits at nearly $10^{500}$, although there could be still more.

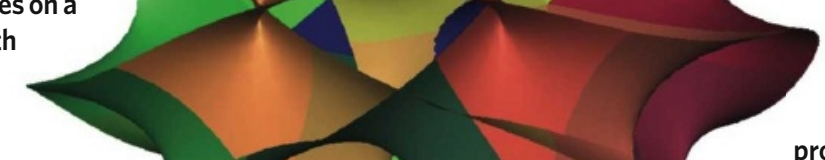

In the 1980s and 1990s, theorists had hoped that there might be a vacuum 'selection process' - a natural

extension of string theory that would select a single vacuum resembling our Universe. But

A cosmos folded up (simulation —not actual size).

fundamental state of the Universe. ours, whereas others might have six or eight or ten. The strengths of those forces, the masses and number of fundamental particles, and even @2008 Nature PublishingGroup 
